\title{
Utilisation \\ de la statistique descriptive en géotechnique
}

M. CASSAN

S.A. Fondasol Études BP 767

84035 Avignon Cedex 3
Actuellement, le choix des valeurs des caractéristiques géotechniques est relativement subjectif. Il n'en est plus ainsi si l'on soumet les résultats des mesures à une analyse statistique. Cette communication présente quelques mêthodes statistiques simples qui conduisent à une évaluation rationnelle de ces caractéristiques et qui prennent en compte les incertitudes et la dispersion des mesures grâce aux notions d'intervalles de confiance pour les caractéristiques indépendantes, et d'ellipses de confiance pour les caractéristiques liées. Mais ces méthodes permettent également d'apprécier l'homogénéité d'un site, par exemple avec le procédé de l'analyse des variances. Nous en avons illustré les approches théoriques par l'examen de quelques cas réels. L'intérêt éyident que présentent ces méthodes devrait inciter tous les ingénieurs géotechniciens à les utiliser très largement et à les généraliser dans leurs études.

Mots clés : histogramme, moyenne, variance, ajustement, loi normale, Student, Snedecor, intervalle de confiance. ellipse de confiance, homogénéité.
NDLE: Les discussions sur cet article sont acceptées jusqu'au $1^{\text {er }}$ août 2001.
So far, selecting values for geotechnical data has been rather subjective. One solution is to submit the results to a statistical analysis. This paper presents some simple statistical methods, which lead to a rational evaluation of the data, taking into account the uncertainties and the dispersion of the measures due to confidence interval, for independent data, and confidence ellipses, for linked data. These methods also provide an appreciation of the homogeneity of the studied site, for example by analyzing the variance. These theoretical methods are illustrated with concrete examples.

The evident interest aroused by the statistical methods should motivate the geotechnical engineering community to integrate them in their studies on an casual basis.

Key words : histogram, average, variance, adjustment, normal law, Student, Snedecor, confidence interval, confidence ellipse, homogeneity. 


\section{Considérations générales}

Dans le cas général, la démarche intellectuelle de l'ingénieur, quelle que soit sa spécialité, est essentiellement déterministe et pour l'ingénieur géotechnicien elle s'articule selon un organigramme dont l'aboutissement est un calcul et un dimensionnement, opérations, elles aussi, particulièrement déterministes.

Sur cet organigramme (Fig. 1) on constate qu'à partir des données fournies par les reconnaissances géologiques et géotechniques, le travail de l'ingénieur est d'abord un travail d'analyse qui est relativement facile, puis un travail de synthèse qui est de loin le plus difficile car il doit déboucher d'une part sur une stratigraphie représentative du site étudié et d'autre part sur l'attribution, à chacune des couches définies dans cette stratigraphie, d'une valeur unique des caractéristiques géotechniques qui y ont été mesurées.

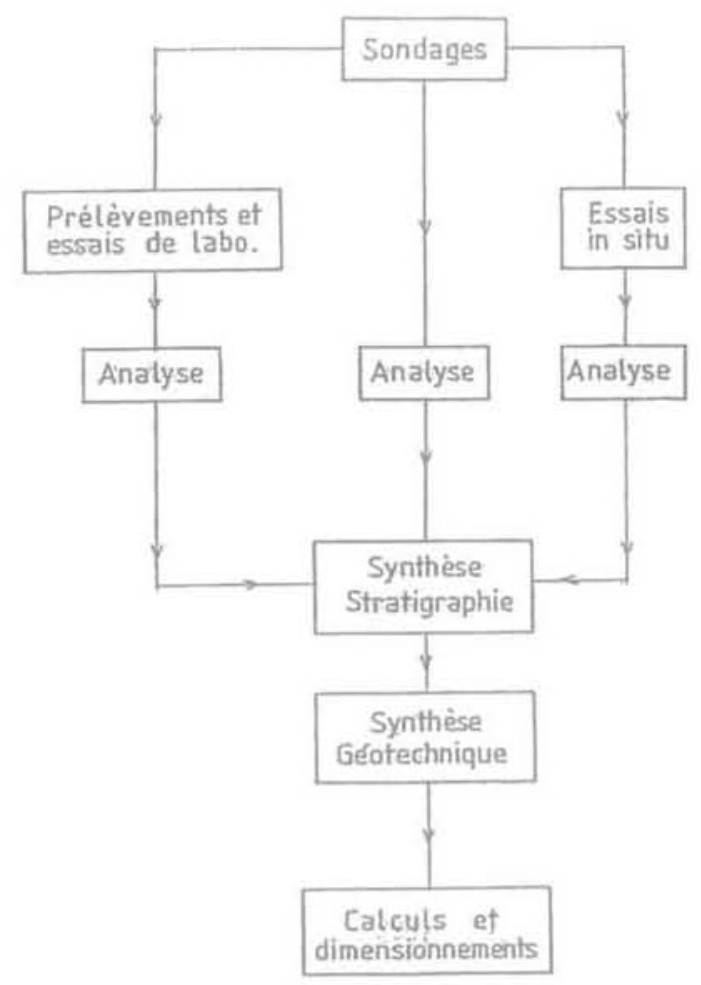

FG. 1 Organigramme d'une étude géotechnique. Flow chart of a geotechnical campaign.

C'est ce dernier point qui s'avère très délicat car il se trouve à la limite de l'objectivité et de la subjectivité. Or, en matière scientifique, la subjectivité ne devrait pas exister. Dans ce contexte, après un premier examen critique des données géotechniques, le traitement statistique de ces données apparait comme un outil extrêmement précieux, susceptible de conférer à la décision de l'ingénieur une plus grande objectivité, l'objectivité totale dans ce domaine étant évidemment très difficile à atteindre sinon impossible.

Mais il faut être très vigilant car, pour que l'outil statistique soit utilisé le plus fréquemment possible et qu'il entre de façon définitive dans le mode de pensée du géotechnicien, il faut qu'il soit simple et maniable. C'est pourquoi nous estimons qu'il est dangereux de brûler les étapes et d'aborder directement le problème avec des théories trop complexes et des mathématiques trop sophistiquées qui, pour réjouissantes qu'elles soient pour l'esprit, risquent de s'avérer dissuasives et d'éloigner définitivement la statistique de la géotechnique.

La présente communication a pour objet d'exposer comment on peut, en un premier temps, envisager l'utilisation de méthodes statistiques simples pour évaluer les caractéristiques mécaniques à attribuer à chaque couche stratigraphique et éventuellement pour apprécier l'homogénéité d'un site.

\section{2}

\section{Synthèse stratigraphique et synthèse géotechnique}

\section{1}

\section{Synthèse stratigraphique}

La synthèse stratigraphique consiste à déterminer une stratification telle que, dans chacune des couches lithologiques mises en évidence par les sondages, les variations des caractéristiques géotechniques mesurées puissent être considérées comme « apparemment » indépendantes des variations spatiales. Elle est établie à partir de :

- l'analyse des coupes de forages et des échantillons : - l'analyse des diagraphies éventuelles (très important); - l'analyse des résultats des essais in situ.

En effet, il ne suffit pas de se fier à la seule stratification lithologique car, dans une couche de nature lithologique ou géologique constante, il peut $y$ avoir des niveaux de caractéristiques géotechniques différentes, comme par exemple des strates d'altération ou des zones superficielles de moindre compacité. Nous donnons plus loin un exemple de cette dernière particularité dans des alluvions fluviatiles. Des phénomènes analogues peuvent également se produire aux interfaces de sols fins (argiles ou limons par exemple) et de terrains granulaires (sable, graves et galets) dans lesquels la limite entre les deux formations peut s'avérer confuse dans les caisses d'échantillons et où seules les diagraphies et les essais in situ permettent de trancher sans ambiguité.

\section{2}

\section{Synthèse géotechnique}

La synthèse stratigraphique ayant permis de délimiter un certain nombre de couches de même nature et apparemment homogènes, le problème consiste à attribuer à une couche donnée une valeur unique de chacune des caractéristiques géotechniques que l'on y a mesurées, ce qui permet de rejoindre le processus déterministe pour la poursuite de l'étude. C'est là qu'intervient le traitement statistique des données. pour lequel on est amené à distinguer deux catégories de caractéristiques géotechniques :

- les caractéristiques indépendantes, directement mesurables: 
- les caractéristiques liées, déterminées simultanément à partir d'un même essai.

Dans la première catégorie, on trouve en particulier: - les paramètres d'identification tels que la teneur en eau, les limites d'Atterberg, le poids volumique :

- quelques caractéristiques mécaniques mesurées en laboratoire comme la résistance à la compression simple, la résistance à la traction (essai brésilien) la pression de préconsolidation, l'indice de compression (Cc), le coefficient de consolidation (c) :

- la plupart des essais in situ, tels que l'essai pressiométrique avec la pression limite nette et le module de déformation, le scissomètre avec la cohésion non drainée, les termes de pointe et de frottement pénétrométriques, le SPT.

Dans la deuxième catégorie se situent essentiellement la cohésion et l'angle de frottement interne qui peuvent être déterminés soit en laboratoire par essais triaxiaux ou essais de cisaillement à la boite, soit in situ à l'aide d'essais au phicomètre.

\section{3}

\section{Méthodes d'estimation statistique des caractéristiques géotechniques}

\section{1}

\section{Caractéristiques indépendantes directement mesurables}

\section{3at:}

\section{Étude de la distribution des mesures}

Dans chaque couche apparemment homogène, on considère, dans leur ensemble, toutes les mesures $x$ du paramètre étudié, tous forages et toutes profondeurs confondues.

Les théories statistiques nous apprennent qu'il existe des tests permettant de s'assurer du caractère aléatoire d'une série d'observations successives. Je citerai pour mémoire, deux tests non paramétriques assez simples et assez rapides qui sont le test des suites et le test des signes [7]. Mais l'utilisation préalable de ces tests ne paraît pas fondamentale dès lors que l'on cherche à ajuster les observations sur une loi statistique connue, car si cet ajustement est possible c'est que la distribution présente effectivement un caractère aléatoire.

On opère donc de la façon suivante :

a) établissement de l'histogramme des n mesures groupées en $k$ classes $(k \geq 5)$ de même $\ll$ largeur n c'est-à-dire telles que, $x_{1}$ et $x_{j+1}$ désignant les valeurs de $x$ qui limitent la classe $j$, les différences $\left[x_{j+1}-x_{j}\right]$ soient constantes et égales à $\frac{x_{r}-x_{1}}{k}$.

Si n désigne la population de la classe $j$, on peut prendre pour ordonnée de cette classe la valeur n, ou, comme le font les statisticiens, la fréquence :

$$
\mathrm{f}_{j}=\frac{\mathrm{n}_{1}}{\sum \mathrm{n}_{j}}=\frac{\mathrm{n}_{1}}{\mathrm{n}}
$$

b) calcul de la moyenne empirique $\bar{x}$ de la population, de sa variance empirique $\mathrm{s}^{2}$, de sa variance estimée $\mathrm{s}^{2}$ et de son coefficient de variation CV :

$$
\begin{array}{ll}
\bar{x}=\frac{1}{n} \sum x_{1} & s^{* 2}=\frac{\sum\left(x_{i}-\bar{x}\right)^{2}}{n} \\
s^{2}=\frac{\sum\left(x_{1}-\bar{x}\right)^{2}}{n-1} & C V=\frac{s}{\bar{x}}
\end{array}
$$

La moyenne arithmétique $\overline{\mathrm{x}}$ est un estimateur de la vraie valeur $\mathrm{M}$ de la moyenne du paramètre étudié, et s? l'estimateur de la vraie valeur $\Sigma^{2}$ de sa variance. Le coefficient de variation caractérise la dispersion des mesures et de la distribution de x. C'est un paramètre très important qui peut, presque à lui seul, donner une idée de la qualité d'une reconnaissance ou du caractère particulier du terrain rencontré puisque l'analyse d'un très grand nombre de résultats a permis de définir des plages de valeurs types de ce coefficient de variation pour diverses caractéristiques géotechniques [6 et 14]. Lorsqu'on s'éloigne de ces plages, il faut donc se poser des questions. Mais c'est l'étude de l'histogramme expérimental qui permettra d'aller plus loin dans la dérnarche statistique.

c) Ajustement de l'histogramme sur une loi de probabilité connue

- Si l'histogramme présente une tendance centrale, c'est-à-dire si l'on voit apparaître une certaine symétrie par rapport à la moyenne ou à la médiane de la distribution, on pourra chercher à ajuster cette distribution sur la loi normale (ou de Laplace-Gauss), ce qui est très facile surtout avec les moyens informatiques actuels.

On utilise pour cela la méthode de la droite de Henry qui consiste à porter en abscisse arithmétique la limite supérieure $s_{\text {, }}$ de la classe j et en ordonnée gaussienne les fréquences cumulées correspondantes. Rappelons que l'échelle gaussienne est une échelle graduée en $\mathrm{u}=\mathrm{f}^{-1}(\mathrm{y})$ où $\mathrm{f}(\mathrm{u})$ est l'expression de la loi de LaplaceGauss en coordonnées réduites:

$$
y=f(u)=\frac{1}{\sqrt{2 \pi}} \int_{-}^{u} e^{-\frac{u^{2}}{2}} d u
$$

Si les points ainsi obtenus sont alignés, c'est que la distribution suit effectivement la loi de Laplace.

On peut alors vérifier la probabilité que l'on a de se tromper si l'on ne retient pas la normalité de la distribution en utilisant le test du $\chi^{2}$ pour $n>30$ (échantillons importants) ou le test de Shapiro-Wilk pour $n<30$ (petits échantillons) ces deux tests étant bien connus des statisticiens (cf. bibliographie, en particulier 7 et 14).

- Si l'histogramme présente une dissymétrie avec étalement vers la droite, on pourra chercher à l'ajuster sur une loi log-normale dans laquelle c'est le logarithme népérien des mesures qui suit la loi de Laplace-Gauss. On utilise là aussi la méthode de la droite de Henry, mais dans un système de coordonnées gausso-logarithmique.

Les paramètres $\mu$ et $\sigma^{2}$ estimateurs respectifs de l'espérance mathématique et de la variance théorique de la loi log normale ajustée, sont liés aux moyenne et variance empiriques par les relations : 


$$
\begin{aligned}
& \mu=\ln \frac{\bar{x}^{2}}{\sqrt{\bar{x}^{2}+s^{* 2}}} \\
& \sigma^{2}=\ln \left(1+\frac{s^{* 2}}{\bar{x}^{2}}\right)
\end{aligned}
$$

- Si ces tentatives d'ajustement s'avèrent infructueuses, on essayera une loi a bêta n plus complexe. Dans ce cas, il faudra soumettre la loi choisie à l'un des tests d'acceptation évoqués précédemment.

L'estimation de la caractéristique étudiée se fait ensuite à partir de la loi de distribution retenue en utilisant la théorie de l'échantillonnage, comme nous allons le voir.

\section{3,102}

\section{Estimation à partir de la loi normale}

\section{a) Notion d'intervalle de confiance}

On admet, dans ce qui suit, que l'ensemble des n mesures réalisées constitue un échantillon unique de taille n prélevé dans une population infinie à distribution normale dont la moyenne vraie $\mathrm{M}$ et la variance vraie $\Sigma^{2}$ sont inconnues. Toutefois, on en connait deux estimateurs $\bar{x}$ et $s^{2}$, qui sont eux-mèmes des variables aléatoires alors que $\mathrm{M}$ et $\Sigma^{2}$, qui resteront toujours inconnues, sont de nature parfaitement déterministe. La théorie de l'échantillonnage permet alors de définir pour $M$ et $\Sigma^{2}$ des intervalles de confiance dépendants de $\bar{x}$, de $s^{2}$ et de $n$, ces intervalles de confiance étant eux-mêmes des variables aléatoires qui ont une certaine probabilité $\mathrm{P}$ de recouvrir $\mathrm{M}$ et $\Sigma^{2}$.

Bien que $\mathrm{M}$ et $\Sigma^{2}$ ne soient pas aléatoires, l'usage admet, par simplification, que l'on dise que $\mathrm{M}$ ou $\Sigma^{2}$ ont la probabilité P d'être compris entre les bornes inférieures I et supérieure S de l'intervalle de confiance et l'on écrira : Prob $\left[\mathrm{I} \leq \mathrm{M}\right.$ (ou $\left.\left.\Sigma^{2}\right) \leq \mathrm{S}\right]=\mathrm{P}$.

Cette relation correspond à un intervalle bilatéral, mais on peut également avoir des intervalles de confiance unilatéraux tels que :

$$
\begin{aligned}
& \text { Prob }\left[M \text { ou } \Sigma^{2} \leq S\right]=P \\
& \text { Prob }\left[M \text { ou } \Sigma^{2} \geq I\right]=P
\end{aligned}
$$

\section{b) Intervalle de confiance de la moyenne}

On démontre que la variable aléatoire $\bar{x}$ a même espérance mathématique, c'est-à-dire mème moyenne $\mathrm{M}$, que la population totale infinie, alors que sa variance n'est pas la variance estimée $s^{2}$, mais :

$$
s_{\bar{x}}^{2}=\frac{s^{2}}{n}
$$

On démontre également que la quantité

$$
t=\frac{\bar{x}-M}{s_{\bar{x}}}=\frac{(\bar{x}-M) \sqrt{n}}{s}
$$

suit une loi de probabilité appelée loi de Student dont la courbe de densité est une courbe symétrique en cloche dans laquelle intervient un paramètre v appelé degré de liberté et qui, pour chaque valeur de $v$, donne la valeur $t_{\alpha}$ ayant la probabilité $\alpha=1-\mathrm{P}$ d'être dépassée (Fig, 2).

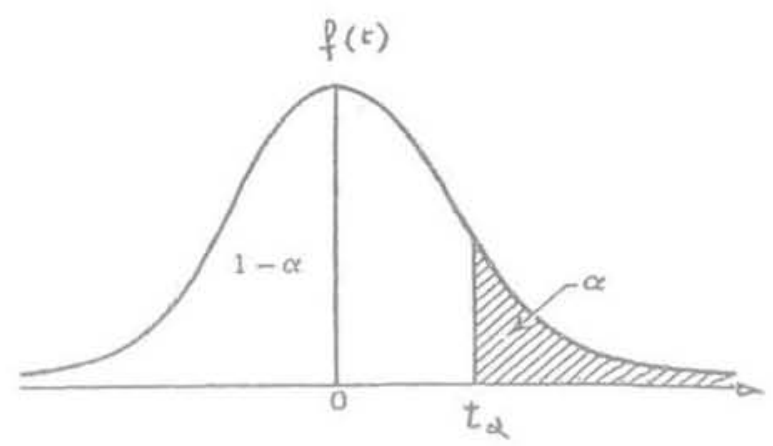

FG. 2 Loi de Student. Intervalle unilatéral. Student probability density fonction. Unilateral interval.

Le degré de liberté est égal à la population de l'échantillon diminuée du nombre de paramètre que l'on estime, c'est-à-dire, pour la loi normale, un seul paramètre (la moyenne) d'où $\mathrm{v}=\mathrm{n}-1$.

Dans le cas de la figure 2 qui correspond à un intervalle de confiance unilatéral, et en se référant à l'expression de $t$, on a :

$$
\operatorname{Prob}\left[M \geq \bar{x}-t_{\alpha} \frac{s}{\sqrt{n}}\right]=1-\alpha=P
$$

Dans le cas de l'intervalle de confiance bilatéral (Fig. 3), on écrit :

$$
\begin{aligned}
& \operatorname{Prob}\left[\overline{\mathrm{x}}-\mathrm{t}_{\alpha / 2} \frac{\mathrm{s}}{\sqrt{\mathrm{n}}} \leq \mathrm{M} \leq \overline{\mathrm{x}}+\mathrm{t}_{\alpha / 2} \frac{\mathrm{s}}{\sqrt{2}}\right] \\
& =1-\alpha=\mathrm{P}
\end{aligned}
$$

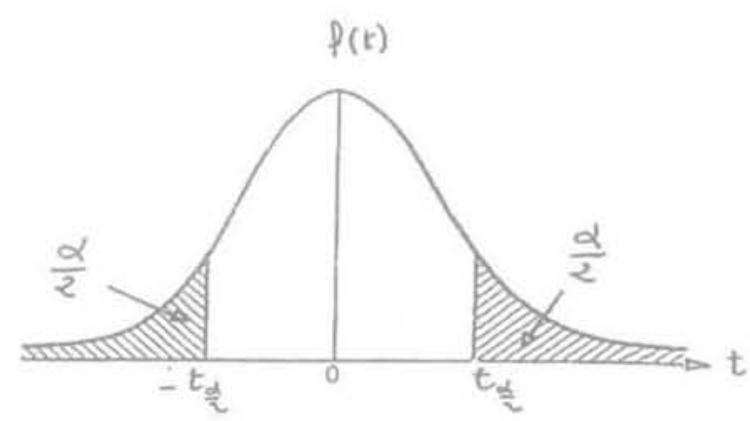

HG. 3 Loi de Student. Intervalle bilatéral, Student probability density fonction. Bilateral interval.

L'intervalle de confiance au seuil de probabilité $\mathrm{P}=1-\alpha$ est donc, pour $\mathrm{n}-1$ degrés de liberté :

$$
[\mathrm{I}, \mathrm{S}]=\overline{\mathrm{x}} \pm \mathrm{t}_{\alpha / 2} \frac{\mathrm{s}}{\sqrt{\mathrm{n}}}
$$

\section{c) Intervalle de confiance de l'écart-type}

- Lorsque $n \leq 30$, on démontre que le rapport $(n-1)$ $\frac{s^{2}}{\Sigma^{2}}$ est une variable aléatoire qui suit la loi probabiliste de Pearson, dite loi de $\chi^{2}$, et dont le nombre de degrés 
de liberté est dans le cas présent égal à n-1, puisqu'on n'estime qu'un seul paramètre.

L'intervalle bilatéral de l'écart-type au niveau de probabilité $\mathrm{P}=1-\alpha$ est alors (Fig. 4)

$$
s \sqrt{\frac{n-1}{\chi_{\frac{\alpha}{2}}^{2}}} \leq \Sigma \leq s \sqrt{\frac{n-1}{\chi_{1}^{2} \frac{\alpha}{2}}}
$$

$\chi_{\alpha}^{2}$ étant la valeur de $\chi^{2}$ ayant la probabilité $\alpha$ d'être dépassée.

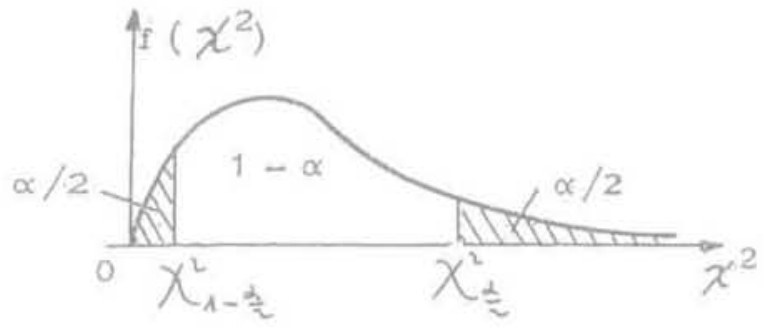

F10.4 Loi du $\chi^{2}$. Intervalle bilatéral pour $\mathrm{n}<30$ $\chi^{2}$ probability density fonction. Bilateral interval for $\mathrm{n}<30$

Les intervalles de confiance unilatéraux sont de même :

$$
\begin{gathered}
0 \leq \Sigma \leq s \sqrt{\frac{n-1}{\chi^{2}, \alpha}} \\
\Sigma \geq s \sqrt{\frac{n-1}{\chi_{\alpha}^{2}}}
\end{gathered}
$$

- Lorsque $n>30$ la distribution de l'écart-type s est à peu près normale. La moyenne vraie reste égale à $\mathrm{M}$ et la variance est voisine de $\frac{\mathrm{s}^{2}}{2 \mathrm{n}}$.

L'expression $\sqrt{2 \chi^{2}}-\sqrt{2 n-1}$ suit la loi de LaplaceGauss réduite d'espérance mathématique 0 et de variance 1.

En désignant par u la variable normale centrée réduite ayant la probabilité $\alpha$ d'être dépassée, on obtient comme intervalle de confiance bilatéral (Fig. 5):

$$
\begin{aligned}
& \operatorname{Prob}\left[\mathrm{s}\left(1-\frac{\mathrm{u}_{\alpha / 2}}{\sqrt{2 \mathrm{n}}}\right) \leq \Sigma \leq \mathrm{s}\left(1+\frac{\mathrm{u}_{\alpha / 2}}{\sqrt{2 \mathrm{n}}}\right)\right. \\
& {[\mathrm{I}, \mathrm{S}]=\mathrm{S}\left(1 \pm \frac{\mathrm{u}_{\alpha / 2}}{\sqrt{2 \mathrm{n}}}\right)}
\end{aligned}
$$

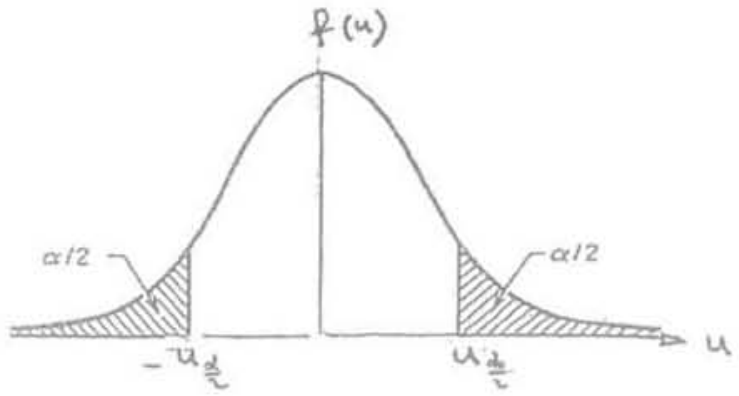

FG,5 Loi de Laplace-Gauss. Intervalle bilatéral pour $\Sigma(n>30)$.

Laplace-Gauss probability density fonction. Bilateral interval for $\Sigma(n>30)$.

\section{d) Choix de la caractéristique étudiée}

A ce stade de l'analyse statistique, la logique voudrait que l'on utilise les résultats précédents pour définir un modèle de sol dans lequel la caractéristique géotechnique étudiée suivrait une loi normale dont les paramètres seraient estimés à partir des intervalles de confiance. Cette estimation devant toujours aller dans le sens de la sécurité, s'il s'agit d'évaluer une caractéristique de résistance, comme par exemple la pression limite nette, la cohésion non drainée ou le nombre $N$ de l'essai de pénétration du carottier (SPT), on pourrait adopter une loi de distribution dont la moyenne $\mathrm{M}^{\prime}$ serait égale à la limite inférieure de son intervalle confiance et l'écart-type $\Sigma$ 'à la limite supérieure de son intervalle de confiance:

$$
\begin{aligned}
& M^{\prime}=\bar{x}-t_{\alpha / 2} \frac{s}{\sqrt{n}} \\
& \Sigma^{\prime}=s \sqrt{\frac{n-1}{\chi^{2} \alpha / 2}} \quad(n<30) \\
& \Sigma^{\prime}=s\left(1+\frac{u_{\alpha / 2}}{\sqrt{2 n}}\right) \quad(n>30)
\end{aligned}
$$

On pourrait alors adopter comme valeur de la caractéristique géotechnique $\mathrm{x}$ étudiée, la valeur $\mathrm{x}_{8}$ telle que (Fig. 6): Prob $\left(x \geq x_{p}\right)=P=1-\beta$.

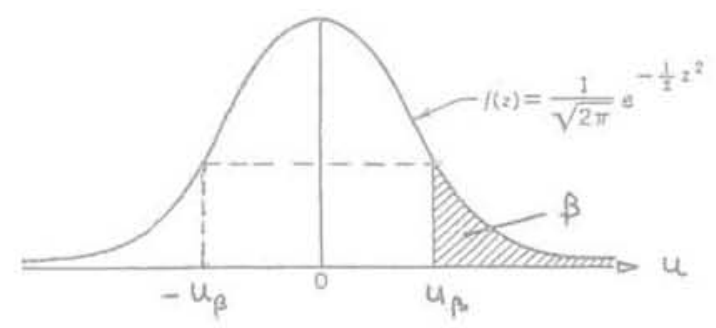

FG.6 Loi de Laplace-Gauss. Laplace-Gauss probability density fonction.

C'est-à-dire en passant à la variable centrée réduite u

$\operatorname{Prob}\left(\mathrm{u}>-\mathrm{u}_{\beta}\right)=1-\operatorname{Prob}\left(\mathrm{u}>\mathrm{u}_{\beta}\right)=1-\int_{u_{\beta}}^{+\infty} \mathrm{f}(\mathrm{u}) \mathrm{du}$ où f(u) est la fonction de densité de probabilité de la loi normale centrée réduite. 
Si l'on prend par exemple $\beta=5 \%$, on $\mathrm{a}_{\beta}=1,64$, donc $x_{\beta}=M^{\prime}+\left(-u_{\beta}\right) \Sigma^{\prime}=M^{\prime}-1,64 \Sigma^{\prime}$

C'est à partir de cette valeur que l'on pourrait rejoindre le raisonnement déterministe. Toutefois cette méthode s'avère pénalisante, et conduit à des probabilités de ruine non négligeables de l'ordre de $10^{-2}$ à $10^{-3}$, parce qu'on n'a pas fait intervenir la notion de longueur d'auto-corrélation et la réduction de variance.

Sans chercher à aborder pour l'instant la probabilité de ruine, nous proposons, pour la pratique courante, une méthode beaucoup plus simple qui, depuis vingt ans, nous a donné entière satisfaction et quil consiste à adopter comme caractéristique de calcul la limite inférieure de l'intervalle de confiance de la moyenne:

$$
x_{c}=\bar{x}-t_{\alpha_{2}} \frac{s}{\sqrt{n}}
$$

Lorsque le choix sécurisant n'est pas une estimation par défaut, mais au contraire une estimation par excès de la caractéristique étudiée, comme par exemple un module de déformation permettant de mettre en évidence un tassement différentiel dû à une zone plus raide, on adoptera la limite supérieure de l'intervalle de confiance de la moyenne.

\section{1:3.}

\section{Estimation à partir de la loi log normale}

On sait que, dans cette loi, c'est le logarithme de la variable aléatoire qui suit la loi normale. On pourrait donc envisager d'appliquer la méthode précédente, c'est-à-dire d'adopter comme valeur du paramètre étudié celle qui correspond à la limite la plus sécurisante de l'intervalle de confiance de la moyenne de son logarithme.

Nous avons eu l'occasion de procéder ainsi mais les valeurs obtenues étaient extrêmement pénalisantes. C'est pourquoi, maintenant (et cela depuis plusieurs années) nous adoptons simplement la valeur modale $\mathrm{x}_{\mathrm{m}}$ de la distribution ajustée (Fig. 7).

Si les paramètres de cette distribution ajustée sont respectivement $\mu$ et $\sigma$, la variable aléatoire $U=\frac{1_{n} x-\mu}{\sigma}$ suit la loi normale centrée réduite et on a :

$$
x_{m}=\exp \left(\mu-\sigma^{2}\right)
$$

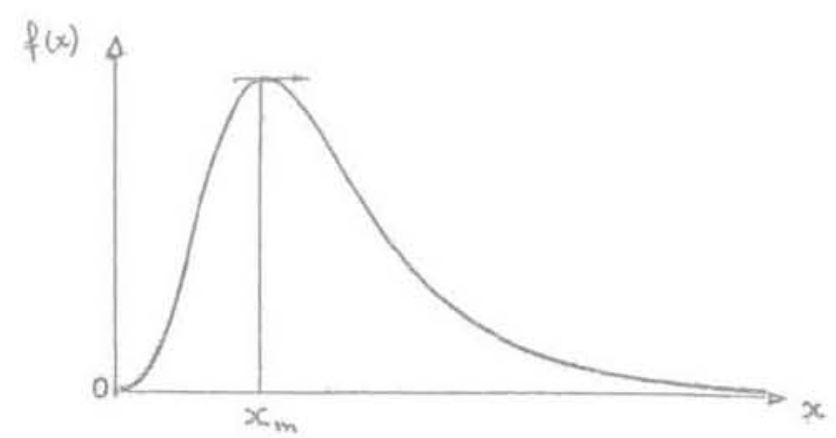

FIG.7 Loi log-normale. Log-normal probability density fonction.

\section{Caractéristiques liées à détermination simultanée}

Il s'agit là essentiellement de la détermination de la cohésion effective c' et de l'angle de frottement interne $\varphi^{\prime}$ en laboratoire, ou d'une évaluation in situ au phicomètre de ces paramètres que l’on désigne alors par $\varphi$ et c, pour éviter toute confusion.

Dans tous ces essais, on mesure deux variables x et y qui doivent théoriquement être liées par une relation fonctionnelle linéaire, $\mathrm{y}=\mathrm{A}+\mathrm{Bx}$, dans laquelle $\mathrm{A}$ et $\mathrm{B}$ sont des paramètres fonctions de $c^{\prime}$ et $\varphi$ ' que l'on cherche à déterminer.

La définition des variables $\mathrm{x}$ et $\mathrm{y}$ est donnée dans le tableau suivant :

\begin{tabular}{l|c|c} 
Essais & $x$ & $y$ \\
Triaxial & $\frac{\sigma_{1}^{\prime}+\sigma_{2}^{\prime}}{2}$ & $\frac{\sigma_{1}^{\prime}-\sigma_{2}^{\prime}}{2}$ \\
\hline Cisaillement & $\sigma$ & $\tau^{\prime}$ \\
\hline Phicomètre & $\sigma$ & $\tau$ \\
\hline
\end{tabular}

La méthode d'interprétation statistique de ces essais consiste à porter sur un même graphique pour chaque couche de terrain l'ensemble des couples $(x, y)$ tous forages, toutes profondeurs, tous échantillons confondus et toutes éprouvettes, et d'effectuer les calculs suivants :

1) Calcul de la droite des moindres carrés dont le coefficient de régression $\mathrm{R}$ doit être supérieur à 0,85 . L'équation de cette droite est $\bar{y}=a+b x$, où a et b sont les estimateurs de $A$ et $B$.

2) Calcul des intervalles de confiance de A et B :

$$
[A]=a \pm t_{\frac{\alpha}{2}, n-2} s \sqrt{\frac{1}{n}+\frac{\bar{x}^{2}}{\sum_{1}^{n}\left(x_{1}-\bar{x}\right)^{2}}}
$$

$$
[B]=b \pm t_{\frac{a}{2}, n-2} \frac{s}{\sqrt{\sum_{i}^{n}\left(x_{i}-\bar{x}\right)^{2}}}
$$

avec :

$t_{\alpha / 2} n$-2variable de Student à $n-2$ degrés de liberté pour un seuil de probabilité $1-\alpha$.

$$
\mathrm{s}^{2}=\frac{1}{\mathrm{n}-2} \sum_{1}^{n}\left(\mathrm{y}_{1}-\overline{\mathrm{y}}\right)^{2}
$$

oủ $n$ est le nombre de couples $\left(x_{i}, y_{i}\right)$ et $y_{1}-\hat{y}$ est l'écart des observations par rapport à la droite des moindres carrés, $\bar{x}$ étant la valeur moyenne des $\mathrm{x}$.

On peut alors déterminer des estimateurs de A et de $B$ sécurisants, en prenant par exemple les valeurs minimales des intervalles de confiance, ou en admettant comme vraie la valeur de a ou celle de b et en ne prenant en considération que l'intervalle de confiance de l'autre. 
Mais la méthode la plus rigoureuse consiste à traiter simultanément $\mathrm{A}$ et $\mathrm{B}$ en faisant appel à la notion d'ellipse de confiance, c'est-à-dire à l'ellipse quí a la probabilité $\mathrm{P}$ de contenir les vraies valeurs de $\mathrm{A}$ et $\mathrm{B}$ lorsque A est porté en abscisse et $\mathrm{B}$ en ordonnée.

En pratique, on fait le changement de variable:

$\mathrm{X}=\mathrm{A}-\mathrm{a}$

$\mathrm{Y}=\mathrm{B}-\mathrm{b}$

$X$ et $Y$ représentent donc les écarts entre les vraies valeurs de $\mathrm{A}$ et $\mathrm{B}$ inconnus et leurs estimateurs a et $\mathrm{b}$ connus. L'équation de cette ellipse est alors :

$$
X^{2}+\beta X Y+\alpha Y^{2}-\gamma=0
$$

avec:

$$
\begin{aligned}
& \alpha=\frac{1}{n} \sum_{1}^{n} x_{1}^{2} \\
& \beta=\frac{2}{n} \sum_{1}^{n} x_{i} \\
& \gamma=\frac{2 s^{2} F_{p}}{n}
\end{aligned}
$$

$F_{0}$ est la valeur d'une variable aléatoire $\mathrm{F}$ qui suit la loi de Snedecor à $\mathrm{v}_{1}=2$ et $\mathrm{v}_{2}=\mathrm{n}-2$ degrés de liberté et telle que Prob $\left(\mathrm{F}<\mathrm{F}_{\mathrm{p}}\right)=\mathrm{P}$. Cette valeur est évidemment donnée par des tables numériques.

Par analogie avec le cas des caractéristiques indépendantes, on évaluera $A$ et $B$ en se plaçant sur l'ellipse elle-mème en des points que l'on aura jugé à la fois sécurisants et physiquement réalistes.

On en déduit alors les caractéristiques de cisaillement :

$\begin{array}{llll}\text { Essai triaxial } & \varphi^{\prime}=\operatorname{Arcsin} B & \text { et } & C^{\prime}=\frac{A}{\cos \varphi^{\prime}} \\ \text { Cisaillement } & \varphi^{\prime}=\operatorname{Arctg} B & \text { et } & C^{\prime}=A \\ \text { à la boite } & & & \\ \text { Phicomètre } & \varphi_{1}=\operatorname{ArctgB} & \text { et } & C_{1}=A\end{array}$

\section{4}

\section{Étude de l'homogénéité d'un site}

L'appréciation de l'homogénèité d'un site reste quelque peu subjective mais la statistique descriptive peut lui conférer une certaine objectivité.

Nous présentons ci-après trois méthodes que nous avons eu l'occasion d'utiliser et qui ne sont valables que pour des caractéristiques indépendantes dont la distribution est soit normale soit ramenée à la normale par introduction d'une fonction auxiliaire (loi log-normale, par exemple).

\section{4.}

\section{Méthode approchée}

Cette méthode est basée sur la comparaison de deux moyennes et de deux variances et utilise un test d'acceptation.

Considérons donc un site sur lequel on a réalisé $\mathrm{r}$ forages dans chacun desquels on dispose de $n_{i}$ mesures du paramètre que l'on cherche à évaluer $(1 \leq i \leq r)$, ce qui porte le nombre total de mesures sur le site

à $N=\sum_{i}^{r} n_{i}$ soit alors :

$\overline{\mathrm{x}}=$ moyenne empirique des $\mathrm{N}$ mesures

$\mathrm{s}^{2}=$ variance estimée des $\mathrm{N}$ mesures

$\bar{x}_{i}=$ moyenne empirique des $n_{1}$ mesures réalisées dans le $\mathrm{i}$ - ième forage

$\mathrm{s}^{2}=$ Variance estimée des $\mathrm{n}$ mesures réalisées dans le i-ième forage

\section{Alatín}

Homogénéité des variances

On compare successivement les variances estimées $\mathrm{s}^{2}$ de chaque forage à la variance estimée $\mathrm{s}^{2}$ de la popu-

lation totale tous forages confondus.
$\quad$ Pour cela, on forme la variable aléatoire $F_{\mathrm{l}}=\frac{\mathrm{s}^{2}}{\mathrm{~s}^{2}}$ qui suit la loi de Snedecor à $v_{1}=N-1$ et $v_{2}=n_{1}-1$ degrés de liberté.

L'hypothèse de l'égalité des variances sera acceptée au niveau de probabilité $\alpha$ si :

$$
\frac{1}{F_{\alpha / 2, n_{1}-1, N-1}} \leq F_{1} \leq F_{\alpha / 2, n_{1}-1, N-1}
$$

ce qui équivaut à accepter l'hypothèse nulle lorsque celle-ci est vraie avec une probabilité 1 - $\alpha$, c'est-à-dire que le risque de se tromper en rejetant l'hypothèse est égal à $\alpha$.

Une autre méthode plus simple mais qui n'est possible que si le nombre de mesures est le même dans tous les forages, est le test de Cochran qui permet de tester simultanément l'égalité de toutes les variances obtenues:

$\mathrm{s}_{1}^{2}=\mathrm{s}_{2}^{2}=\ldots \ldots \ldots . \mathrm{s}^{2}$,

pour cela on forme le rapport :

$$
y=\frac{\text { maximum de } s^{2}}{\sum_{i}^{f} s_{i}^{2}}
$$

L'hypothèse d'égalité sera acceptée au seuil de probabilité $\alpha$ si $g \leq g_{\alpha^{\prime}} g_{\alpha}$ étant donné par une table numérique établie pour $\alpha=0,05$ et $\alpha=0,01$ et qui est fonction de $n$ (nombre de mesures par forages) et $\mathrm{r}$ (nombre de forages).

\section{aits \\ Homogénéité des moyennes}

On compare successivement les moyennes $\bar{x}_{i}$ de

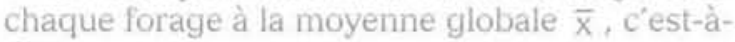
dire

que l'on cherche à voir si la différence $\bar{x}_{i}-\bar{x}$ peut être considérée comme nulle avec une probabilité satisfaisante. Le test précédent ayant permis de décider si l'on est en droit d'admettre ou de rejeter l'égalité des 
variances, on doit donc considérer ces deux cas, dans chacun desquels les variances restent toujours inconnues.

a) Variances égales

On forme la variable aléatoire

$$
t_{i}^{\prime}=\frac{\bar{x}_{1}-\bar{x}}{s \sqrt{\frac{1}{n_{i}}+\frac{1}{N}}}
$$

qui suit la loi de Student à $v_{1}=n_{1}+N-2$ degrés de liberté et dans laquelle $\mathrm{s}^{2}$ est la variance commune à tous les forages.

Le domaine d'acceptation de l'égalité des moyennes du forage i et de celle de l'ensemble des forages est alors :

$$
-t_{\alpha / 2^{\prime} \text { wi }} \leq t_{1} \leq-t_{\alpha 2^{\prime} \text { wi }}
$$

\section{b) Variances différentes}

Sur le plan théorique et purement mathématique il n'est pas possible, dans ce cas, d'utiliser un test paramétrique de décision. Toutefois, selon A.H. Bowker et G.J. Lieberman [2] on peut définir une variable statistique t' qui ne suit qu'approximativement la loi de Student lorsque les moyennes sont égales :

$$
t_{i}^{\prime}=\frac{\bar{x}_{i}-\bar{x}}{\sqrt{\frac{s^{2}}{n_{i}}+\frac{s^{2}}{N}}}
$$

qui suit la loi de Student à v degrés de liberté avec :

$$
v_{1}=\frac{\left(\frac{s^{2}}{n_{i}}\right)^{2}+\left(\frac{s^{2}}{N}\right)^{2}}{\frac{1}{n_{1}+1}\left(\frac{s^{2}}{n_{1}}\right)^{2}+\frac{1}{N+1}\left(\frac{s^{2}}{N}\right)^{2}}-2
$$

La valeur numérique de $v$ ainsi obtenue n'est en général pas entière; on adoptera alors l'entier le plus voisin.

Les critères de rejet de l'hypothèse $\bar{x}_{i}-\bar{x}=0$ au niveau de probabilité $\alpha$ sont alors les suivants :

$$
\begin{aligned}
& \left|t^{\prime}\right| \geq t_{\alpha z^{\prime}} v \text { : les deux moyennes sont significative- } \\
& \text { ment distinctes; } \\
& t_{1}^{\prime} \geq t_{\alpha, v}: \text { les deux moyennes sont distinctes avec } \\
& \bar{x}_{i}>\bar{x} ; \\
& t_{1}^{\prime} \leq-t_{\alpha v v} ; \text { les deux moyennes sont distinctes avec } \\
& \bar{x}_{1}<\bar{x} .
\end{aligned}
$$

La probabilité $\alpha$ signifie que l'on a $\alpha$ chance de se tromper si l'on rejette l'hypothèse nulle alors qu'elle est vraie.

\section{Méthode de l'analyse des variances}

Cette méthode est plus rigoureuse que la précédente et permet d'apprécier globalement si les mesures effectuées dans tous les forages appartiennent à la même population et si les écarts constatés entre forages résultent du hasard, chaque forage étant considéré comme un échantillon de l'ensemble de la formation. Les hypothèses de base sont : distribution suivant la loi normale et même nombre n de mesures par forages. Le nombre total de mesures dans la formation étudiée est donc $\mathrm{N}=\mathrm{nr}$.

La méthode consiste alors à évaluer de deux façons différentes la variance $\mathrm{s}^{2}$ de la population totale dont la moyenne est $\bar{x}$.

- Évaluation de $\mathrm{s}^{2}$ à partir des termes relatifs à chaque forage

$$
\begin{aligned}
& s_{i}^{2}=\frac{1}{r} \sum_{i=1}^{i=r}\left[\frac{1}{n-1} \sum_{j=1}^{n n}\left(x_{i j}-\bar{x}_{i}\right)^{2}\right] \\
& =\frac{1}{N-r} \sum_{i=1}^{j=r} \sum_{j=1}^{j=n}\left(x_{i j}-\bar{x}_{i}\right)^{2}
\end{aligned}
$$

$x_{1 j}$ désignant la j-ième mesure dans le i-ième forage - Évaluation de $s^{2}$ à partir des moyennes de chaque forage :

$$
s_{2}^{2}=\frac{n}{r-1} \sum_{i=1}^{i=r}\left(\bar{x}_{i}-\bar{x}\right)^{2}
$$

Le rapport $F=\frac{s_{2}^{2}}{s_{1}^{2}}$ doit être voisin de 1 et son écart avec l'unité ne doit pas être significatif. Or F est une variable aléatoire qui suit la loi de Snedecor à $v_{1}=r-1$ et $v_{2}=\mathrm{N}-\mathrm{r}$ degrés de liberté.

La condition d'acceptation de l'hypothèse au seuil de probabilité $\alpha$ est alors: $\mathrm{F} \leq \mathrm{F}_{\alpha,-1, N-r}$

S'il n'en est pas ainsi, l'hypothèse doit être rejetée et le terrain peut être considéré comme hétérogène.

Dans la pratique, on présente les résultats sous forme d'un tableau dans lequel la variance est égale à la somme des carrés divisée par le nombre de degrés de liberté.

\begin{tabular}{l|c|c|c}
$\begin{array}{l}\text { Sources } \\
\text { de variation }\end{array}$ & $\begin{array}{c}\text { Somme } \\
\text { des carrés }\end{array}$ & $\begin{array}{c}\text { Degrés } \\
\text { de liberté }\end{array}$ & Variance \\
\hline Totalité des mesures & $\sum_{i=1}^{i=r} \sum_{j=1}^{j=n}\left(x_{i j}-\bar{x}\right)^{2}$ & $N-1$ & $s^{2}$ \\
\hline En forage & $\sum_{i=1}^{i=1} \sum_{j=1}^{j=n}\left(x_{i j}-\bar{x}_{1}\right)^{2}$ & $N-r$ & $s^{2}$ \\
\hline Entre forages & $n \sum_{i=1}^{i=t}\left(\bar{x}_{i}-\bar{x}\right)^{2}$ & $r-1$ & $s_{2}^{2}$ \\
\hline
\end{tabular}




\section{Méthode de Tukey}

La méthode de Tukey [2] consiste à chercher si l'intervalle de confiance $\overline{\mathrm{x}}_{\max }-\overline{\mathrm{x}}_{\min } \pm \mathrm{K}$ recouvre zéro avec une probabilité $1-\alpha$.

S'il est ainsi, tous les couples $\left(\bar{x}_{p}, \bar{x}_{q}\right)$, où $p$ et $q \leq r$, sont tels que :

$$
\overline{\mathrm{x}}_{\max }-\overline{\mathrm{x}}_{\min }-\mathrm{K} \leq \overline{\mathrm{x}}_{\mathrm{p}}-\overline{\mathrm{x}}_{\mathrm{q}} \leq \overline{\mathrm{x}}_{\max }-\overline{\mathrm{x}}_{\min }+\mathrm{K}
$$

On peut alors admettre que $\bar{x}_{p}=\bar{x}_{q}$ avec la probabilité $1-\alpha$, et que le site est homogène.

S'il n'en est pas ainsi, la différence entre $\bar{x}_{\operatorname{mix}}$ et $\bar{x}_{\text {min }}$ doit être considérée comme significative et il faut reprendre le calcul pour d'autres couples de moyennes.

Le facteur $k$ est donné par la relation:

$$
\mathrm{k}=\mathrm{k}_{\circ} \sqrt{\frac{\mathrm{s}_{1}^{2}}{\mathrm{n}}} \text {. }
$$

où $\mathrm{k}_{0}$ est fourni par des tables en fonction de r et de $v=N-r$ et pour $\alpha=0,05$ et $\alpha=0,01$ [2].

On voit que cette méthode nécessite l'établissement du tableau d'analyse de la variance.

La méthode de Tukey peut également être utilisée pour préciser les forages qui ont conduit, si tel est le cas, à un rejet de l'hypothèse d'homogénéité par la méthode d'analyse de la variance.

\section{5}

\section{Examen de cas réels}

\section{1}

\section{Détermination des caractéristiques géotechniques}

\section{S.1.}

\section{TGV Méditerranée (1995-1997), Viaduc sur le Rhône à Avignon}

La reconnaissance lithologique a fait apparaître quatre couches principales numérotées de haut en bas de 1 à 4. L'examen des profils pressiométriques a toutefois mis en évidence des différences de compacité dans la couche des alluvions grossières du Rhône selon que l'on se trouve sur les berges, c'est-à-dire à terre, ou dans le lit du fleuve. De plus, dans le lit du fleuve lui même, on a noté un très important affaiblissement des caractéristiques pressiométriques $\left(E_{M}\right.$ et $\left.p_{1}-p_{o}\right)$ dans la partie supérieure des alluvions grossières, c'est-à-dire précisément dans la zone qui, avant la stabilisation du lit par les barrages de la Compagnie nationale du Rhône, était le siège d'affouillements importants, pouvant atteindre 8 à 10 mètres de profondeur. Nous avons donc été amenés à diviser cette formation alluvionnaire en trois sous-couches.
Dans le substratum marneux rencontré au-dessous des alluvions, les modules pressiométriques mesurés à terre sont légèrement inférieurs à ceux obtenus dans le lit du Rhône, mais cela pour des raisons purement géologiques. Nous avons donc considéré, dans ce substratum, deux couches géotechniquement différentes. En définitive, alors que la lithologie et la géologie donnaient quatre couches différentes, la synthèse stratigraphique nous a amenés à en définir sept.

La figure 8 montre les histogrammes des modules pressiométriques dans trois de ces couches avec les droites de Henry correspondantes.

Dans les alluvions à terre (Fig, 8a) l'histogramme est grosso modo symétrique et les points représentatifs des fréquences cumulées en coordonnées gausso-arithmétiques sont très correctement alignés, avec un coefficient de régression linéaire de 0,995.

La distribution de $\mathrm{E}_{\mathrm{M}}$ est donc normale avec pour la loi ajustée :

$$
\overline{\mathrm{x}}=40 \mathrm{Mpa} \quad \mathrm{s}=19 \mathrm{Mpa} \quad \mathrm{n}=55
$$

Pour un niveau de probabilité $P=0,95$ soit $\alpha=1-P=0,05$ et $n-1=54$ degrés de liberté, la table de Student donne $t_{\alpha / 2}=2,005$, La limite inférieure de l'intervalle de confiance de la moyenne donné par la formule (10) est alors :

$$
I=40-2,005 \frac{19}{7,42}=34,86 \mathrm{MPa}
$$

Nous avons donc retenu pour cette formation : $E_{\mathrm{M}}=35 \mathrm{Mpa}$.

Les histogrammes des figures $8 \mathrm{~b}$ et $8 \mathrm{c}$, concernant respectivement la partie supérieure des alluvions grossières et le substratum situés dans le lit du Rhône, présentent tous deux une asymétrie avec étalement vers la droite.

Les courbes de fréquences cumulées en coordonnées gausso-logarithmique montrent que les distributions sont log-normales avec :

- alluvions grossières $3 \mathrm{a}: \mu=2,88$ et $\sigma=0,77$

d'où la valeur modale (formule 11) :

$\mathrm{X}_{\mathrm{m}}=9,8 \approx 10 \mathrm{MPa}$

- substratum : $\mu=6,11$ et $\sigma=0,62$

d'oủ la valeur modale: $\mathrm{X}_{\mathrm{m}}=307 \approx 300 \mathrm{MPa}$

Nous avons donc adopté :

- alluvions grossières lâches : $E_{M}=10 \mathrm{Mpa}$; - substratum dans le fleuve : $\mathrm{E}_{\mathrm{M}}=300 \mathrm{Mpa}$.

En opérant ainsi pour toutes les couches de la synthèse stratigraphique, nous avons obtenu le tableau ciaprès qui nous a permis de poursuivre l'étude des fondations de façon déterministe. 

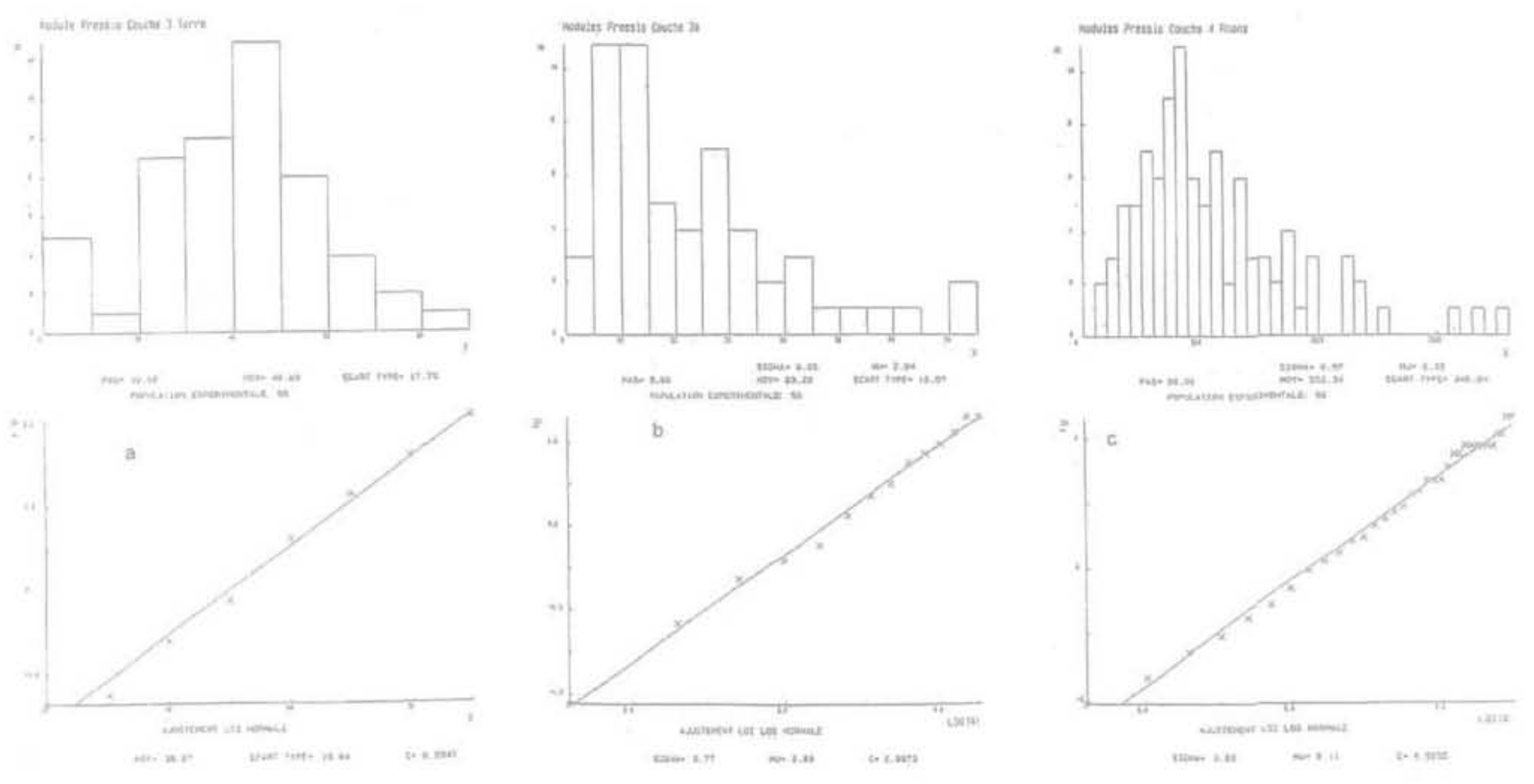

FIG.8 TGV Méditerranée, viaduc d'Avignon. Distribution des modules pressiométriques.

TGV Méditerranée (High Speed Train Track), bridge in Avignon. Distribution of the pressurmeter modulus.

\begin{tabular}{|c|c|c|c|}
\hline Désignation des sols & Nature & $E_{M}(\mathrm{MPa})$ & $P_{1}-P_{o}(\mathrm{MPa})$ \\
\hline 1 & Limons et terrains meubles de couverture & 2,2 & 0,3 \\
\hline 2 & Sables gris, quelques graviers & 11,3 & 1,0 \\
\hline 3 & Alluvions grossières à terre & 35,0 & 4,0 \\
\hline $3 a$ & Alluvions grossières Rhöne & 10,0 & 2,0 \\
\hline $3 \mathrm{~b}$ & Alluvions grossières Rhône & 50,0 & 5,4 \\
\hline \multirow[t]{3}{*}{4} & Substratum Rhône & 300,0 & 7,0 \\
\hline & Substratum terre & 220,0 & 7,0 \\
\hline & Substratum global & 270,0 & 7.0 \\
\hline
\end{tabular}

\section{B.t.2}

\section{TGV Méditerranée. Déblai du pâtis}

Il s'agit d'une grande tranchée qui entaille une butte de sable compact et de marne silteuse dans le département de la Drôme (plaine de Marsanne). Une première campagne de reconnaissance a été réalisée en 1995 au cours de laquelle, conformément à notre marché, nous avons réalisé dans chacune des deux formations, deux essais triaxiaux CU + U et un essai de cisaillement consolidé drainé à la boîte. C'était vraiment peu pour un tel terrassement!
En 1997, la SNCF nous a demandé une reconnaissance complémentaire avec, à nouveau, deux essais triaxiaux $\mathrm{CU}+\mathrm{U}$ et un essai à la boîte $\mathrm{CD}$ dans chaque couche.

La figure 9 représente les résultats que nous avons obtenus dans les marnes argileuses grises en groupant les deux campagnes avec tous les couples de points ( $s^{\prime}$, $\left.t^{\prime}\right)$ ou $\left(\sigma^{\prime}, \tau^{\prime}\right)$, tous échantillons et toutes profondeurs confondus. Nous remarquons sur le graphe supérieur de la figure (9a) que les points $\left(s^{\prime}, t^{\prime}\right)$ sont remarquablement alignés avec un coefficient de régression de 0,996, ce qui montre l'homogénéité des deux campagnes. 


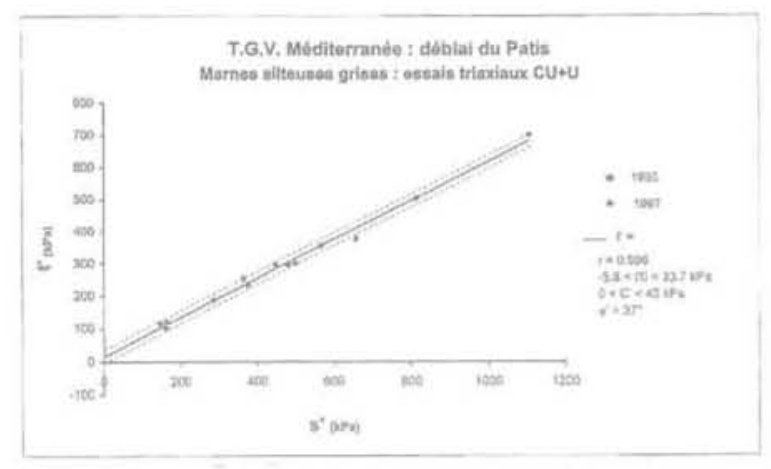

Momes siteusen grises "essain frioxitux

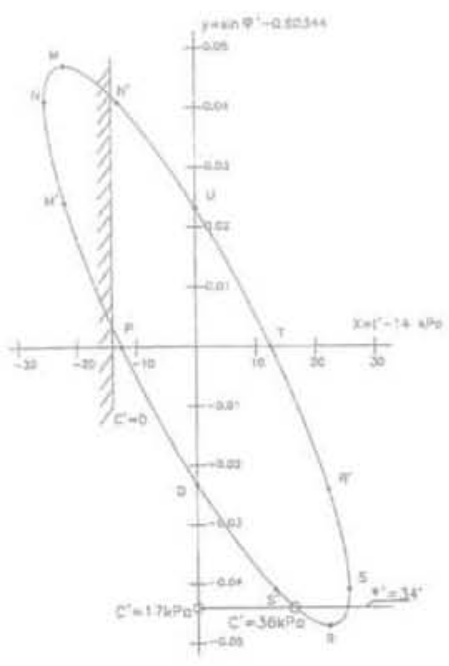

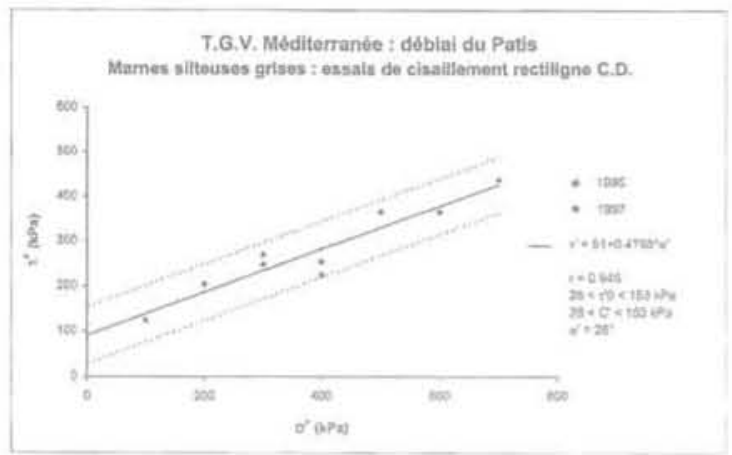

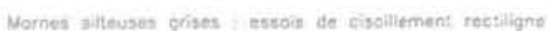

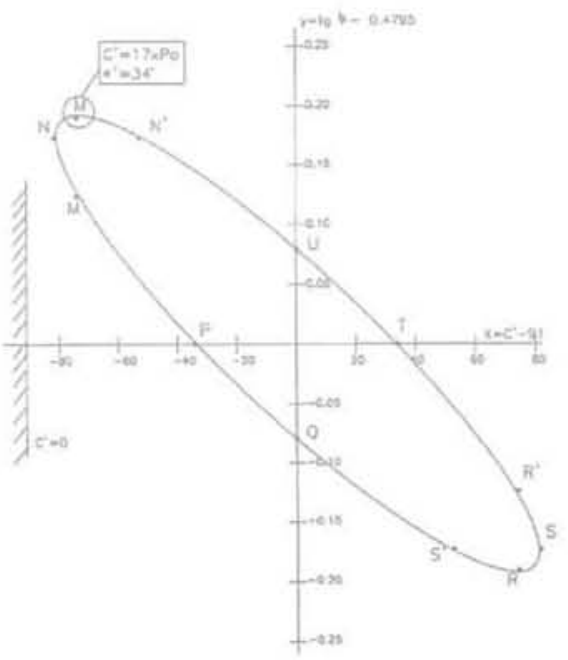

Les deux droites en pointillés parallèles à la droite des moindres carrés donnent l'intervalle de confiance à $95 \%$ de l'ordonnée à l'origine de la droite de régression (formule 12). Mais, comme indiqué précédemment, il est plus rigoureux dans ce type d'estimation d'utiliser la notion d'ellipse de confiance et de se placer sur le contour le plus sécurisant de cette ellipse.

La figure 9a (inférieure) montre qu'il est prudent de chercher une valeur de ' $\varphi^{\prime}$ et $C^{\prime}$ vers le bas de la courbe, pour laquelle nous avons adopté un niveau de probabilité de $95 \%$. Nous pouvons donc prendre: $\varphi^{\prime}=34^{\circ}$ et $\mathrm{c}^{\prime}=36 \mathrm{kPa}$ pour autant que ce résultat soit compatible avec l'essai de cisaillement à la boite qui conduit, chose surprenante, à des valeurs de $\varphi^{\prime}$ significativement plus faibles que celles obtenues dans les essais triaxiaux.

Nous voyons ainsi sur la figure $9 \mathrm{~b}$ (inférieure) que le seul point qui corresponde à $\varphi^{\prime}=34^{\circ}$ est le point de contact $\mathrm{M}$ de la tangente horizontale supérieure à l'ellipse. En revanche, ce point conduit à $c^{\prime}=17 \mathrm{kPa}$. Le point qui correspond à $\mathrm{c}^{\prime}=36 \mathrm{kPa}$ est voisin de $\mathrm{N}^{\prime}$ et conduit à $\varphi^{\prime}=33^{\circ}$ valeur peu différente de 34 degrés.

On peut donc admettre qu'il y a comptabilité (un peu limite certes) entre les deux essais, en ce qui concerne l'angle de frottement interne, mais pas en ce qui concerne la cohésion. Par mesure de prudence, on a adopté $c^{\prime}=17 \mathrm{kPa}$, mais on a conservé $\varphi^{\prime}=34^{\circ}$.

Sur la figure 10, relative aux sables compacts, nous voyons que la linéarité des points représentatifs des essais triaxiaux est également remarquable avec un coefficient de régression linéaire de 0,993, à opposer à celui des essais de cisaillement à la boite qui n'est que de 0,843 .

S'agissant d'un sable, nous avons résolument adopté une cohésion nulle tout à fait compatible avec les deux types d'essai, et les ellipses de coefficience au niveau de probabilité de $95 \%$ montrent qu'il y a également compatibilité des deux types d'essai pour $\varphi^{\prime}=43^{\circ}$. Nous avons donc adopté dans les sables $\varphi^{\prime}=43^{\circ}$ et $c^{\prime}=0$.

\section{1 .3}

\section{Tramway de Strasbourg}

Il s'agissait d'évaluer les caractéristiques de résistance au cisaillement $\varphi^{\prime}$ et $c^{\prime}$ des alluvions du Rhin, qui ne sont évidemment pas carottables.

La difficulté a été contournée en procédant à des mesures in situ au phicomètre, appareil qui permet, à tout niveau, d'appliquer une série de pressions radiales $\sigma_{i}$ sur les parois d'un forage et, pour chacune de ces pressions, d'exercer un effort de traction vertical coaxial au forage jusqu'à la rupture, effort qui correspond, sur la paroi du forage, à une contrainte de cisaillement $\tau_{i}$.

Ainsi, à chaque niveau, on dispose d'un certain nombre de couples (5 à 8 environ) de valeurs de $\sigma$ et $\tau$ qui permettent de tracer la droite de Coulomb et d'en déduire des valeurs de $\varphi_{i}$ et $c_{i}$ peu différentes, en milieu granulaire, de $\varphi^{\prime}$ et $c^{\prime}$, selon l'inventeur de l'appareil, G. Philipponnat. 

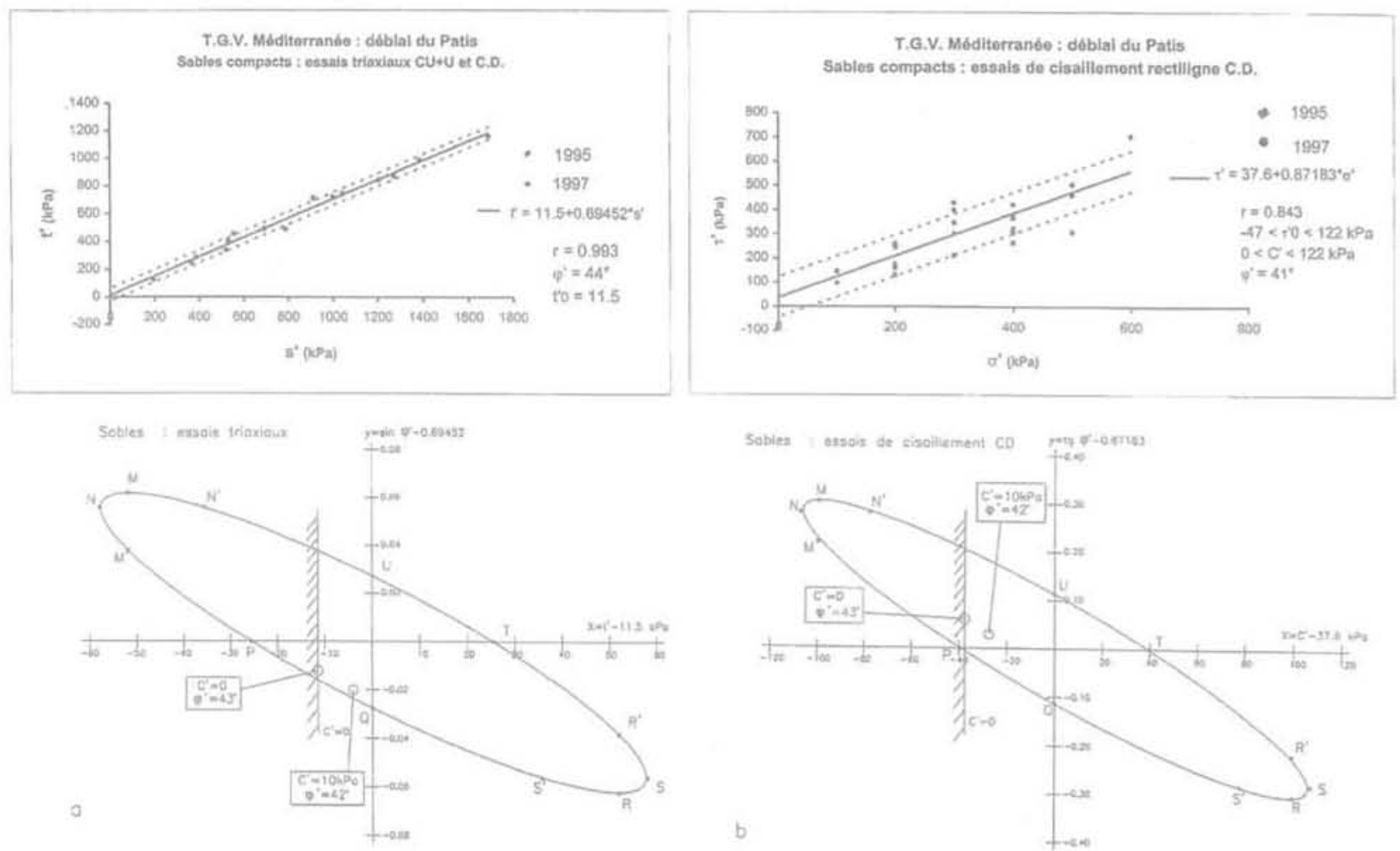

FG, 10 TGV Méditerranée. Déblai du pâtis. Évaluation de $\varphi^{\prime}$ et $\mathrm{C}^{\prime}$. Ellipse de confiance. TGV Méditerranée (High Speed Train Track). Patis Excavation. Evaluation of $\varphi^{\prime}$ et $C^{\prime}$. Confidence ellipse.

Dans le cas présent, on a réalisé de tels essais tous les 1,60 mètre environ jusqu'à 10 mètres de profondeur. Ces essais ont donné des résultats très dispersés avec :

$$
23^{\circ} \leq \varphi_{1} \leq 41^{\circ} \text { et } 0 \leq \mathrm{C}_{1} \leq 72 \mathrm{kPa}
$$

Nous avons donc porté sur le mème graphique (Fig. 11a) l'ensemble des couples $(\sigma, \tau)$ mesurés tous forages et toutes profondeurs confondues, soit 90 couples. La droite des moindres carrés du nuage de points a conduit à un coefficient de régression de 0,912 ce qui est tout à fait correct et justifie cette approche, et a donné un angle de 34 degrés avec une cohésion de $16 \mathrm{kPa}$.

Nous n'étions pas d'accord pour attribuer à cette formation essentiellement granulaire une quelconque cohésion. mais le conseil du maitre d'œuvre, s'appuyant sur le fait qu'on n'avait pu justifier les déformations des parois du métro de Lyon qu'en faisant intervenir une cohésion dans les alluvions du Rhône, voulait absolument en prendre une dans les alluvions du Rhin, qui ne sont pas plus cohérentes que celles du Rhône.

Or l'examen de l'ellipse de confiance a montré qu'avec une cohésion de $10 \mathrm{kPa}$, l'intervalle de confiance de $\varphi^{\prime}$ au niveau de probabilité de $95 \%$ était tel que : $33^{\circ} \leq \varphi \leq 35,5^{\circ}$ $10 \mathrm{kPa}$.

Nous avons donc finalement adopté : $\varphi^{\prime}=34^{\circ}$ et $c^{\prime}=$

Tout s'est très bien comporté, mais nous avons toutefois clairement précisé, dans notre étude, que la cohésion prise en compte ne constituait pas une liaison entre les particules du sol, comme dans une argile, mais résultait d'une imbrication des grains, et que cette soit disant cohésion n'était qu'un artifice dủ à l'extrapolation de la partie linéaire de la courbe $\tau^{\prime}=f\left(\sigma^{\prime}\right)$ pour les faibles valeurs de $\sigma^{\prime}$.

\section{2}

\section{Homogénéité d'un site}

\section{8xal}

\section{Limons argileux de Cagnes-sur-Mer}

Dans ces matériaux relativement cohérents, nous avons réalisé 75 essais au scissomètre qui ont donné des valeurs conventionnelles de la cohésion non drainée et qui ont été répartis sur 5 forages.

Nous avons testé l'homogénéité du site avec la méthode approchèe développée en 4.1 , en comparant les moyennes empiriques obtenues dans chaque forage à la moyenne empirique globale de l'ensemble des mesures. Avec les mêmes notations, nous avons le tableau suivant:

\begin{tabular}{l|r|r|r|r} 
Ne Forages & $\bar{x}_{1}$ & \multicolumn{1}{c|}{$s_{1}^{e}$} & $n_{1}$ & $\frac{s_{i}^{2}}{n_{1}}$ \\
$(\mathrm{kPa})$ & 38 & 121 & 26 & 4,65 \\
\hline SC1 & 43 & 64 & 21 & 3,05 \\
\hline SC2 & 49 & 289 & 20 & 14,45 \\
\hline SC3 & 52 & 9 & 3 & 3,0 \\
\hline SC4 & 35 & 121 & 5 & 24,2 \\
\hline SC5 & & & & \\
\hline
\end{tabular}



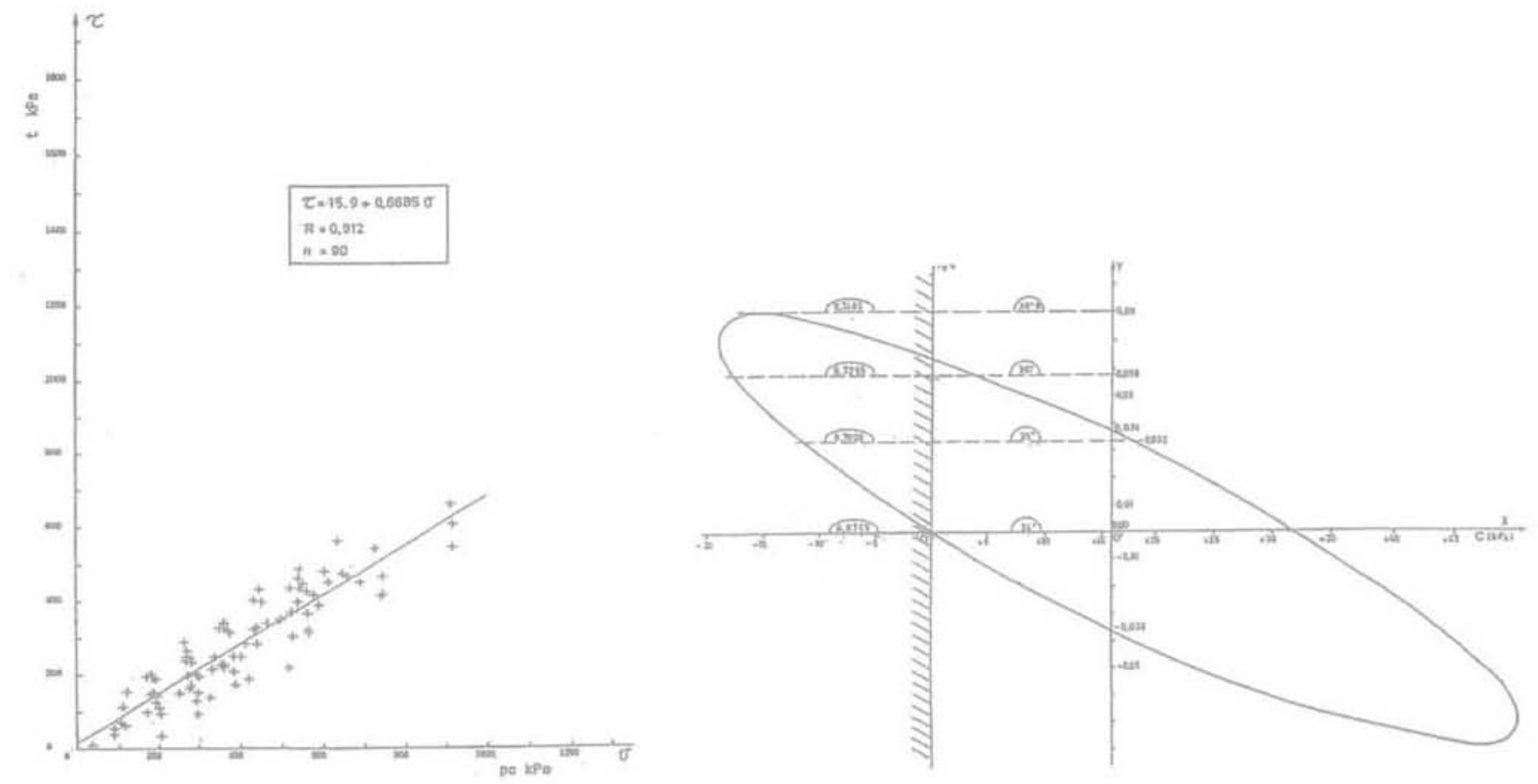

Rstmanow gLoeate

FIG.11 Tramway de Strasbourg. Évaluation de $\varphi_{\text {, }}$ et $C_{\text {, }}$ au phicomètre.

Tramway in Strasbourg. Alluvial deposits of the Rhin River. Evaluation of $\varphi_{1}$ and $C_{1}$ with phicometer.

Nous avons par ailleurs pour la population totale:

$$
\mathrm{N}=75 \quad \overline{\mathrm{x}}=43 \mathrm{kPa} \quad \mathrm{s}^{2}=169 \quad \frac{\mathrm{s}^{2}}{\mathrm{~N}}=2,25
$$

A partir des formules (19) et (20), nous obtenons, tous calculs faits pour $\alpha=0,05$ :

\begin{tabular}{|c|c|c|c|c|}
\hline $\mathrm{N}^{2}$ forage & $t^{\prime}$ & $v$ & $t_{\infty 2 x i}$ & tane \\
\hline $\mathrm{SCl}$ & $-1,896$ & 52 & 2,009 & 1.676 \\
\hline $\mathrm{SC}_{2}$ & 0,00 & 57 & 2,000 & 1,671 \\
\hline $\mathrm{SC} 3$ & 1,466 & 26 & 2,056 & 1,706 \\
\hline $\mathrm{SC} 4$ & 3.928 & 10 & 2,228 & 1.812 \\
\hline $\mathrm{SC} 5$ & $-1,564$ & 5 & 2,570 & 2,015 \\
\hline
\end{tabular}

On constate que seul l'écart entre la moyenne de la population totale et la moyenne obtenue dans le forage SC4 est significatif par valeur inférieure, les autres écarts ne l'étant pas.

On peut dire que la zone de SC4 présente une particularité (affaiblissement ou essais défectueux) alors que le reste du site est homogène.

\section{Gigag}

\section{Marnes argileuses de Palavas-les-Flots}

Ces marnes ont fait l'objet de prélèvements d'échantillons intacts et d'essais de compression simple en laboratoire dans le cadre de l'étude du pont des Quatre-Canaux. Ces essais étaient répartis en quatre forages avec trois essais par forage.

Nous avons utilisé ici la méthode d'analyse de la variance.

Les données des mesures nous ont permis de dresser le tableau ci-après :

\begin{tabular}{l|c|c|c}
\hline NeForage & $x_{n}(\mathrm{MPa})$ & $\bar{x}_{1}(\mathrm{MPa})$ & $s^{7}(\mathrm{MPa})^{2}$ \\
& 0,68 & & \\
SC1 & 0,31 & 0,377 & 0,0762 \\
& 0,14 & & \\
\hline \multirow{3}{*}{ SC4 } & 0,38 & & 0,0016 \\
& 0,31 & 0,357 & \\
\hline \multirow{3}{*}{ SC6 } & 0,38 & & 0,0132 \\
& 0,28 & & \\
\hline \multirow{3}{*}{ SC10 } & 0,48 & 0,413 & 0,0076 \\
& 0,48 & & \\
\hline
\end{tabular}

Le tableau d'analyse des variances est alors le suivant:

\begin{tabular}{l|c|c|c}
\hline $\begin{array}{l}\text { Source } \\
\text { de variation }\end{array}$ & $\begin{array}{c}\text { Somme } \\
\text { des carrés }\end{array}$ & $\begin{array}{c}\text { Degrès } \\
\text { de liberté }\end{array}$ & Variances \\
\hline $\begin{array}{l}\text { Totalité } \\
\text { des mesures }\end{array}$ & 0,20307 & 11 & $s^{2}=0,01846$ \\
\hline En forages & 0,1974 & 8 & $s_{1}^{2}=0,02468$ \\
\hline Entre forages & 0,005534 & 3 & $s_{2}^{2}=0,00184$ \\
\hline
\end{tabular}


On en déduit: $F=\frac{s_{2}^{2}}{s^{2}}=0,07455$

Au niveau de probabilité $\alpha=0,05$ on a, d'après la table de Snédécor:

$\mathrm{F}_{\alpha, r-1, N-\mathrm{N}}=\mathrm{F}_{0,05 ; 3 ; 8}=4,07$

Comme F est largement inférieur à 4,07 , le test est accepté et on peut admettre que le site est homogène.

Appliquons, par curiosité, la méthode de Tukey aux moyennes extrêmes :

on a : $\overline{\mathrm{x}}_{\max }=0,413 \mathrm{MPa}$ (forage SC6)

$\overline{\mathrm{x}}_{\min }=0,357 \mathrm{MPa}$ (forage SC4)

Pour $r=4, v=N-r=8, n=3$ et $\alpha=0,05$, les tables de Tukey donnent $k o=4,53$ d'où :

$$
\begin{aligned}
& \mathrm{k}=\mathrm{k}_{\circ} \sqrt{\frac{\mathrm{s}_{1}^{2}}{\mathrm{n}}}=4,53 \sqrt{\frac{0,02468}{3}}=0,41 \\
& \overline{\mathrm{x}}_{\max }-\overline{\mathrm{x}}_{\min } \pm 0,41=-0,35 / 0,47
\end{aligned}
$$

Cet intervalle recouvre largement le zéro, ce qui confirme bien le résultat de l'analyse des variances.

\section{6}

\section{Conclusion}

Nous avons présenté, dans les développements qui précèdent, quelques-uns des outils que la statistique mathématique met à la disposition de l'ingénieur qéotechnicien. Ces outils peuvent paraître, à première vue, d'un emploi compliqué, mais, en fait, leur utilisation, facilitée par des tables numériques ou des logiciels, est relativement simple, comme le montrent les exemples réels cités.

On a pu voir se dégager tout au long de cet exposé les grandes lignes d'une méthodologie d'analyse qui devrait être appliquée à toute reconnaissance géotechnique. Cette méthodologie comprend trois phases: - le dépouillement des résultats au sens le plus large du terme avec la classification des paramètres mesurés par nature de terrain, l'établissement des histogrammes et leur ajustement sur une loi de probabilité connue ;

- la détermination des valeurs de calculs à partir des notions d'intervalle de confiance pour les caractéristiques indépendantes et d'ellipse de confiance pour les caractéristiques liẻes ;

- l'étude de l'homogénéité d'un site par la méthode d'analyse de la variance ou par des méthodes moins sophistiquées utilisant un test d'hypothèse paramétrique double, basé sur la comparaison de deux moyennes empiriques.

Depuis des décennies, dans la grande majorité des cas, les données d'une reconnaissance géotechnique sont traitées par l'ingénieur de façon plus ou moins subjective. Grâce à l'outil statistique, il devient possible, sans faire appel à une mathématique sophistiquée qui pourrait être dissuasive, de conférer à ces choix techniques une certaine objectivité, qui assure la sécurité sans pour autant empiéter sur le jugement et sur l'expérience de l'homme.

Nous ne pouvons donc qu'exhorter les ingénieurs géotechniciens à utiliser systématiquement ces méthodes, qui conduisent à une meilleure appréciation des propriétés d'un sol mais qui permettent également de juger de la qualité d'une expérimentation.

\section{Bibliographie}

Bonitzer J. (1983) - L'emploi des statistiques. Paris. Presses des Ponts et Chaussées.

Bower A.H., Lieberman G.J. (1964) Méthodes statistiques de l'ingénieur. Paris, Dunod.

Calot G. (1964) - Cours de statistiques descriptives. Paris, Dunod.

Calot G. (1967) - Cours de calcul des probabilités. Paris, Dunod.

Cambefort H. (1971) - Introduction ả la géotechnique. Paris, Eyrolles.

Cassan M. (1979) - « Détermination probabiliste des caractéristiques mécaniques des sols $\mathrm{m}$. Conférence ECP, non publiée.

CEA (1979) - Statistique appliquée à l'exploitation des mesures. Paris, Masson (2 tomes).
Favre J.-L. (1980) - « Milieu continu et milleu discontinu: mesure statistique indirecte des paramètres rhéologiques et approche probabiliste de la sécurité n. These de doctorat d'État en sciences physiques, université Pierre et Marie Curie, Paris.

Guy J. (1967) - Eléments de calcul des probabilités et de calcul statistique. Paris, SEDES.

Harr M.E. (1981) - Mécanique des milieux formés de particules. Presses polytechniques romandes.

Labrousse C. (1978) - Statistiques appliquées. Paris, Dunod (3 tomes).

Liorzou A. (1976) - Initiation pratique à la statistique. Paris, Eyrolles.
Lipschutz S. (1979) - Probabilités. Cours et problèmes, Série Schaum. New York, Mc Graw Hill Inc., 1973, Paris.

Magnan J.-P. (1982) - Los méthodes statistiques et probabilistes en mécanique des sols. Paris, Presses des Ponts et chaussées.

Moreau M. Mathieu A. (1979) Statistique appliquée à l'expérimentation. Paris, Eyrolles,

Mothes J. (1967) - Incertitudes et décisions incustrielles. Paris, Dunod.

Reeb G., Fuchs A. (1967) - Statistiques commentées. Paris, Gauthier-Villars.

Spiegel M.R. - Théorie et application de la statistique. Série Schaum, New YorkParis Mc Graw Ill Inc, 1978. 